\title{
TAGUNG
}

\section{EU-Desintegration? Gegenkräfte und Kriseneffekte im europäischen Integrationsprozess}

\author{
Ronny Patz*
}

Die europäische Integration ist eine Geschichte fortdauernder Krisen, aber jede dieser Krisen erscheint im Lichte gängiger Integrationstheorien rückblickend jeweils nur ein Moment des Innehaltens oder eine Chance für weitere Integrationsschritte gewesen zu sein. Wie ist es aber zu bewerten, wenn die europäische Integration keine Einbahnstraße ist, auf der sich die Union der Staaten und Völker zwar mit unterschiedlicher Geschwindigkeit aber doch immer nur in einer Richtung fortbewegt? Was bedeutet es für die Theorie, wenn der Prozess der Integration stattdessen besser als ein mehrdimensionales Tauziehen zu beschreiben ist, ein Tauziehen um die Ausprägung der gemeinsamen Politik in ihrer Breite und Tiefe und tatsächlich auch Rückschritte möglich sind?

Ein solches mehrdimensionales Tauziehen hat dann nicht ein einziges - ideales oder gar visionäres - Entwicklungsziel. Stattdessen bildet sich im kontinuierlichen Spiel von internen und externen Zug- und Gegenkräften ein in seinen politischen Strukturen, Prozessen und Politikfeldern ganz unterschiedlich integriertes Mehrebenensystem heraus, welches auch im Zeitverlauf nicht notwendigerweise in einer Gleichgewichtsposition ankommen muss, aus der heraus es keine weiteren signifikanten Veränderungen mehr geben kann.

Die nun schon mehrere Jahre andauernde Finanz- und Staatsschuldenkrise erscheint ein angemessener Anlass, sich über solche Zugund Gegenkräfte konzeptionell und empirisch bewusst zu werden, nicht zuletzt, da öffentlich

\begin{tabular}{|c|}
\hline $\begin{array}{c}\text { Zug- und Gegenkräfte im } \\
\text { europäischen Integrationsprozess - } \\
\text { Gefahr eines wachsenden } \\
\text { Ungleichgewichts? }\end{array}$ \\
\hline $\begin{array}{l}\text { Wissenschaftliche Tagung des Arbeitsbereichs } \\
\text { Vergleichende Politikwissenschaft und Euro- } \\
\text { päische Integration der Universität Tübingen, des } \\
\text { Lehrstuhls Politik und Regieren in Deutschland und } \\
\text { Europa der Universität Potsdam, des Arbeitskreises } \\
\text { Europäische Integration e.V., des Forum of Fede- } \\
\text { rations und der Stiftung Genshagen. }\end{array}$ \\
\hline $\begin{array}{l}\text { Die Tagung wurde von der Europäischen Kommis- } \\
\text { sion finanziell unterstützt. }\end{array}$ \\
\hline Genshagen, 16.-17. Februar 2012 \\
\hline $\begin{array}{l}\text { Thematische Einleitung: Zug- und Gegenkräfte } \\
\text { im Spiegel verschiedener Theorien }\end{array}$ \\
\hline $\begin{array}{l}\text { Dr. Annegret EPPLER, Universität Tübingen } \\
\text { Dr. Henrik SCHELLER, Universität Potsdam } \\
\text { Prof. Dr. Arne NIEMANN, Universität Mainz } \\
\text { Dr. Claus-Peter CLOSTERMEYER, Vertretung } \\
\text { des Landes Baden-Württemberg beim Bund, Berlin }\end{array}$ \\
\hline $\begin{array}{l}\text { Zug- und Gegenkräfte der europäischen } \\
\text { Integration in der Verfassungs- und der } \\
\text { Erweiterungspolitik }\end{array}$ \\
\hline $\begin{array}{l}\text { Prof. Dr. Matthias NIEDOBITEK, Technische } \\
\text { Universität Chemnitz } \\
\text { Florian ZIEGENBALG, CDU-Fraktion im Land- } \\
\text { tag von Baden-Württemberg, Stuttgart } \\
\text { Dr. Katrin BÖTTGER, Institut für Europäische Po- } \\
\text { litik, Berlin } \\
\text { Dr. Marzenna GUZ-VETTER, Vertretung der Eu- } \\
\text { ropäischen Kommission in Deutschland, Berlin }\end{array}$ \\
\hline
\end{tabular}

* Ronny Patz, Dipl.-Pol., Doktorand, Wirtschafts- und Sozialwissenschaftliche Fakultät, Universität Potsdam; Promotionsstipendiat der Friedrich-Naumann-Stiftung für die Freiheit. 
über sichtbare Schritte der Desintegration - der Austritt Griechenlands aus der Eurozone, der komplette Zerfall der Währungsunion oder der Austritt Großbritanniens aus der Union - diskutiert wird. Auch wird in der Krise sichtbarer denn je, dass die lange angenommene passive Zustimmung der Bevölkerung zur Europäischen Union, der sogenannte ,permissive consensus', nicht unbedingt und für immer vorausgesetzt werden kann. Die Tagung ,Zugund Gegenkräfte im europäischen Integrationsprozess - Gefahr eines wachsenden Ungleichgewichts?" fokussierte aus wissenschaftlicher Perspektive und aus Sicht von Praktikern nicht nur die Zug-, sondern insbesondere auch die Gegenkräfte der europäischen Integration, und nahm sich damit eines in der bisherigen Integrationsforschung wenig beleuchteten Aspekts der Integration an. Die Überwindung des bereits von Wolfgang Wessels und Anne Faber konstatierten ,normativen bias“ der Integrationstheorie ${ }^{1}$ benötigt möglicherweise nicht nur neue oder zumindest erweiterte Kategorien und Konzepte, sondern auch einen speziellen Blick auf die Empirie sowohl auf der Systemebene als auch in den unterschiedlichen Politikfeldern.

Bereits die Diskussionen am ersten Tag der Tagung, eingeleitet und theoretisch unterfüttert von Annegret Eppler, Henrik Scheller, Arne Niemann und Claus-Peter Clostermeyer und moderiert von Reinhold Herber, zeigten, dass es bisher noch kein gemeinsames Verständnis davon gibt, was unter Zug- und Gegenkräften im europäischen Integrationsprozess verstanden werden soll, wie man diese Kräfte analytisch und empirisch fassen kann oder welche Akteure und welche Phänomene betrachtet werden müssen, um zu verstehen oder gar zu erklären, in welche Richtung sich das politische System der Europäischen Union - als Ganzes und in seinen Politikbereichen - zu welchem Zeitpunkt entwickelt. Auch nach der Abschlussdiskussion am Ende des zweiten Tages und einer Reihe durchaus auch kontrovers
Dinner-Speech: Quo vadis Euro - Zerreißt die Finanzkrise die EU?

Dr. Dieter SPÖRI, Minister a.D.; Präsident, Europäische Bewegung Deutschland, Berlin

Leitbilder, Öffentliche Meinung, Parteien:

Kategoriebildung auf der Polity- und der Politics-Ebene

PD Dr. Olaf LEIßE, Universität Jena

Prof. Dr. Hermann SCHMITT, University of Manchester

Mag. Stefan August LÜTGENAU, Foster Europe, Foundation for Strong Regions in Europe, Eisenstadt

Prof. Dr. Werner REUTTER, Freie Universität Berlin

Zug- und Gegenkräfte in der Wirtschafts- und Finanzpolitik und in der Außen- und Sicherheitspolitik

Dr. Daniela SCHWARZER, Stiftung Wissenschaft und Politik, Berlin

Dr. Matthias WOISIN, Finanzbehörde Hamburg Dr. Matthias DEMBINSKI, Hessische Stiftung Friedens- und Konfliktforschung, Frankfurt Main Dr. Barbara KUNZ, Stiftung Genshagen

Zug- und Gegenkräfte in der Umwelt- und der Bildungspolitik

Dr. Annegret EPPLER, Universität Tübingen

Matthias WINZER, Umweltbundesamt Dessau

Dr. Henrik SCHELLER, Universität Potsdam

\section{Moderation der Abschlussdiskussion}

Dr. Reinhold HERBER, Forum of Federations, Ottawa

geführter Diskussionen waren noch eine Reihe dieser Fragen offen und es gab weiterhin unterschiedliche Auffassungen darüber, was letztendlich unabhängige und abhängige Variable(n) sein sollten.

Darüber hinaus wurde deutlich, wie schwer die analytische Trennung und Operationalisierung von Kräften einerseits und ihren Auswirkungen andererseits ist. Auch wurde diskutiert, dass es zur Bestimmung von Zug- und Gegenkräften

1 Anne Faber/Wolfgang Wessels: Die Verfassungskrise der EU als Krise „der“ Integrationstheorie? Plädoyer für eine Neufokussierung der theoretischen Debatte; in: Zeitschrift für Internationale Beziehungen 2/2005, S. 353-359. 
einer Entwicklungsrichtung bedarf. Immer wieder wurde auch über das Verhältnis der Kategorien Zug- und Gegenkräfte zum Komplex Legitimation und Bürgerbeteiligung nachgedacht. Offen blieb zudem, wie sich Zug- und Gegenkräfte in unterschiedlichen Politikfeldern zueinander verhalten und welches Gewicht man bei der Beantwortung dieser Fragen der aktuellen Krise als Sonderfall zuweisen muss. Dies machte deutlich, dass der durch die Tagung eingenommene Blickwinkel auf die Integration - die Analyse von ,Kräften', die hinter bestimmten Prozessen stehen, sowie die explizite Einbeziehung von Desintegrationstendenzen - in Bereiche vorstößt, die durch bisherige Ansätze noch nicht eindeutig erschlossen sind.

\section{Krisen und Kräfte in den Politikfeldern}

Die empirische Relevanz der Diskussion über Zug- und Gegenkräfte war mit Verweis auf die aktuelle ökonomische Krise und die Diskussionen über Desintegrations-Szenarien bereits zu Beginn der Tagung hervorgehoben und insbesondere durch die Dinner Speech von und die Diskussion mit Dieter Spöri beleuchtet worden. Im Vortrag von Olaf Leiße über Leitbilder des Integrationsprozesses wurde zu Beginn des zweiten Tages noch einmal deutlich, dass die aktuelle Krise nicht nur historisch in einer Reihe von bedeutenden politischen Krisen der europäischen Integration, sondern auch in einer Reihe von ökonomischen Krisen globaler Regionen der vergangenen Jahrzehnte steht. Die aktuelle und die vergangenen Krisen der Europäischen Union seien häufig auch Krisen der Kooperation der Mitgliedstaaten und Krisen gemeinsamer Visionen gewesen, wobei ebenso häufig diese Krisen als Ausgangspunkt für neue Visionen dienten, die als Zugkräfte den Integrationsprozess weiter vorantreiben halfen.

Kann man aber so einfach Zug- und Gegenkräfte während und nach einer Krise identifizieren? Nimmt man die letzte große Krise der verfassungspolitischen Integration - das Scheitern der Referenden in Frankreich und den Nie- derlanden zum Vertrag über eine Verfassung für Europa - und schaut sich den Vertrag von Lissabon als Ergebnis dieses Krisenprozesses an, so ist nach der Argumentation im Beitrag von Matthias Niedobitek über Verfassungspolitik unklar, ob es sich dabei nur um eine Konsolidierung des vorherigen Spiels der Kräfte handelt oder ob der Vertrag und seine Interpretation durch den Europäischen Gerichtshof selbst als Zug- und Gegenkraft im Integrationsprozess zu verstehen sind; etwa wenn der Vertrag über die Europäische Union in Art. 48 Abs. 2 sogar ausdrücklich eine mögliche Verringerung der Kompetenzen der Europäischen Union im Zuge zukünftiger Vertragsrevisionen vorsieht.

Florian Ziegenbalg bestätigte diese Einschätzung und verwies auf weitere Ambivalenzen: Einerseits würden seit dem Inkrafttreten des Vertrags von Lissabon nationale und subnationale Parlamente verstärkt europapolitisch aktiv, andererseits zeigten sich gerade im Kontext der Verhandlungen über den Fiskalpakt stark intergouvernementale Dynamiken, in denen die neuen Rechte von Parlamenten und subnationalen Akteuren schnell wieder beschnitten werden können.

Diese Ambivalenz und die Schwierigkeit, Zugund Gegenkräfte in der Ausgestaltung, Veränderung und Wirkung der Verträge der Europäischen Union klar zu benennen, setzte sich in der Betrachtung verschiedener klassischer und neuerer Politikfelder fort: Im Bereich der Finanz- und Wirtschaftspolitik (Daniela Schwar$z e r)$, die durch die Krise im besonderen Fokus der Aufmerksamkeit stehe, seien bedeutsame Integrationsschritte zu beobachten, etwa, was die gestärkte Rolle der Europäischen Zentralbank angeht. Auch die Formulierung des Krisenrechts (zum Beispiel die Schuldenbremsen) könne als Ergebnis von integrationsfreundlichen Zugkräften gewertet werden, aber bereits hier sei unklar, ob dieses Recht überhaupt jemals angewandt werden wird, ob es sich dabei also tatsächlich um eine Vertiefung der Integration handelt. Gleichsam stellte sich die Frage, ob die Stärkung eines „Dunkelmännergre- 
miums" wie der Europäischen Zentralbank (Matthias Woisin) tatsächlich als sinnvoller Integrationsschritt zu bewerten sei.

Auch in anderen Politikbereichen sind ähnliche Dynamiken zu beobachten, die nicht eindeutig als Entwicklungen hin zu oder weg von mehr Integration zu bewerten sind. In der Erweiterungspolitik (Katrin Böttger) könne die Erweiterung der Union um neue Mitgliedstaaten zum einen als Integrationsschritt betrachtet werden, zum anderen könne Erweiterung aber auch eine Gegenkraft gegen mehr Integration sein, da Konsensfindung über zukünftige Entwicklungen in Konstellationen mit deutlich mehr Akteuren erschwert werde. Ähnlich könnten politische Programme wie die Europäische Nachbarschaftspolitik (als Ersatz für eine Beitrittsperspektive) Ausdruck von Kräften gegen mehr Integration und gleichzeitig Motor für weitere Integrationsschritte sein, indem sie die Verhandlungsbasis für vertiefte Kooperation in bestimmten Politikfeldern zwischen der Europäischen Union und ihren Nachbarstaaten bildeten. Dass diese widersprüchlichen Überlegungen auch tatsächlich Entsprechung in den internen Diskussionsprozessen in den EU-Institutionen und den Mitgliedstaaten finden, konnte Marzenna Guz-Vetter bestätigen.

In der Gemeinsamen Sicherheits- und Verteidigungspolitik (Matthias Dembinski) scheinen Zugkräfte, wie die Notwendigkeit von Einsparungen in nationalen Haushalten, eher pragmatischer Natur zu sein, auch wenn der europäische Friedensdiskurs eine wirkmächtige Vision für mehr Integration bereitstellt. Nichtsdestotrotz wirke aber die Vorstellung von nationaler Souveränität als starke Gegenkraft zu mehr Integration, sodass selbst die Schaffung neuer Institutionen wie des Europäischen Auswärtigen Dienstes - zumal unter einer schwachen Leiterin - nicht notwendig als echte Integrationsschritte zu werten seien. Unklar ist, so die Argumentation von Barbara Kunz, inwieweit in einem Politikfeld wie der Außen- und Sicherheitspolitik Zug- und Gegenkräfte zu identifizieren sind, in dem sich das Mehrebenensystem nicht nur über die nationale sowie die EU- und NATO-Ebene ${ }^{2}$ erstreckt, sondern auch das Weltsystem mit seinen machtpolitischen Großregionen und andere außen- und sicherheitspolitische Ordnungsrahmen relevante Größen sind.

Anders als in der Sicherheitspolitik mit offenbar starken Gegenkräften, lasse sich in einem Politikfeld wie der Umweltpolitik (Annegret Eppler, Matthias Winzer) zumindest regulativ eine Dominanz von Zugkräften beobachten; etwa durch den sukzessiven Verlust von Vetopositionen der EU-Mitgliedstaaten und einer großen Zahl von europäischen Gesetzesvorgaben. Dass in einem stark integrierten Politikfeld trotzdem Gegenkräfte wirken, ließe sich hingegen durch eine große Zahl von Vertragsverletzungsverfahren oder dem politischen Widerstand von ökonomisch starken Regionen gegen bestimmte Vorhaben der EU-Ebene erkennen. Aber selbst in einem vertraglich nur sehr schwach integrierten Politikbereich wie der Bildungspolitik (Henrik Scheller) seien Zugkräfte am Werk, sichtbar zum Beispiel wenn der Europäische Gerichtshof die Verträge integrationsfreundlich auslegt und eine Ausweitung der Brüsseler Kompetenzen von der Berufs- hin zur allgemeinen Bildung zustimmt. Die Vorstöße hin zu einer verstärkten Integration im Bildungsbereich können aber im Gegenzug auch Gegenkräfte wecken, die sich in Protestbewegungen, wie zum Beispiel gegen den Bologna-Prozess, materialisieren könnten.

In allen Politikfeldern können also Phänomene beobachtet werden, die als (unterschiedliche) Kräfte im Integrationsprozess oder zumindest als Indikatoren für solche Kräfte dargestellt werden können. Die unterschiedlichen Beiträge machten aber deutlich, dass über die Perspektive und die auszuwählenden Phänomene keine Einigkeit besteht.

\section{Zug- und Gegenkräfte in den ,politics ‘}

Zug- und Gegenkräfte der europäischen Integration lassen sich aber nicht nur in den ausge-

2 North Atlantic Treaty Organization. 
wählten Politikfeldern sondern auch auf der Ebene der Politikprozesse beobachten. So sei die Herausbildung von europäischen Parteienstrukturen zunächst einmal eine Entwicklung, die als Indikator verstärkter Integration gedeutet werden könne. Was aber, so die von Stefan August Lütgenau aufgeworfene Frage, wenn sich europaweite integrationsfeindliche Gruppierungen zusammenfinden, um als vereinte Gegenkräfte - „Parasiten der Integration“ in den Worten von Werner Reutter - auftreten zu können, womöglich unterstützt durch zunehmend europakritische Wähler, die sich von ihren bisherigen Repräsentanten nicht recht vertreten fühlten? Dieses Gefühl der ungenügenden Repräsentation, so die Ergebnisse eines gerade abgeschlossenen Forschungsprojekts, vorgestellt von Hermann Schmitt, bestätigt sich jedoch empirisch nicht. Parlamentarier in den nationalen Parlamenten seien demnach ähnlich europafreundlich oder -skeptisch wie ihre Wähler. Es scheine daher fraglich, inwieweit europaweit organisierte euroskeptische Kräfte tatsächlich die Unterstützung bekommen könnten, die sie benötigten, um Einfluss auf den weiteren Verlauf der Integration nehmen zu können. Möglicherweise ist eine spannendere Frage, aus welcher Richtung solche Gegenkräfte zu erwarten sind, da zum Beispiel beobachtet wird, dass Wähler in den vormals sozialistischen Mitgliedstaaten tendenziell euroskeptischer sind.

\section{Querschnittsfragen und Ausblick}

Während die hier nur kurz zusammengefassten Beiträge der Konferenzteilnehmer zu den unterschiedlichen Politikbereichen und -prozessen einen durch die Tagungsorganisation vorgegebenen Rahmen bildeten, gab es darüber hinaus einen roten Faden in den jeweiligen Diskussionen, der relativ schnell zeigte, dass es eine Reihe von Querschnittsfragestellungen gibt, die durch eine getrennte Betrachtung von Politikbereichen nur schwer zu beantworten sind: Sind zum Beispiel ein Mehr an demokratischen Beteiligungsrechten und eine verstärk- te europäische Öffentlichkeit Zug- oder Gegenkraft im europäischen Integrationsprozess? Wann ist Widerstand gegen politische Entscheidungen auf der EU-Ebene als Gegenkraft zu werten? Welche Rolle spielen klassische Begriffe der Politik, insbesondere jener der Macht, wenn wir nach Ursachen für bestimmte Kräfte suchen und welche Akteure mit welchen Interessen stehen hinter diesen Kräften? Verbirgt sich hinter der Konzeption von Zug- und Gegenkräften nicht bereits eine normative Vorstellung von einem wünschenswerten Endzustand - und was wäre überhaupt ein solcher Endzustand? Wie kann man Zug- und Gegenkräfte beobachten, vor allem in Situationen, in denen es keine nennenswerten Entwicklungen gibt und sich die Frage stellt, ob dies das Ergebnis von gleich starken Kräften oder der Abwesenheit von Kräften ist? Braucht es nicht in letzter Konsequenz auch eine Theorie der (kompletten) Desintegration?

Der noch im Laufe des Jahres 2012 geplante Tagungsband in der AEI-Schriftenreihe wird zeigen, wie die unterschiedlichen Beiträge der Teilnehmerinnen und Teilnehmer diesen Diskussionsprozess und die Querschnittsfragen verarbeiten und ob es gelingt, die bislang noch lose verknüpften Diskussionsstränge über Zugund Gegenkräfte in der europäischen Integration sinnvoll zu vereinen. Spannend wird es auch sein zu sehen, ob sich die bislang noch relativ vage Konzeption und Operationalisierung von Zug- und Gegenkräften konkretisiert. Wenn dies gelingt, könnten diese Tagung und der Sammelband nicht nur die Integrationsforschung bereichern, sondern es könnte vielleicht - wie von einem der teilnehmenden Praktiker gefordert - sogar ein Beitrag dazu geleistet werden, Handlungsempfehlungen für diejenigen zu entwickeln, die Integration nicht nur als einen analytischen Begriff sondern als ein normatives Ziel verstehen, auf das sie, insbesondere in Krisenzeiten, mit möglichst starken Zugkräften hinarbeiten wollen. Aber vielleicht sind solche Erwartungen zu diesem Zeitpunkt der Debatte auch noch etwas zu optimistisch. 